\title{
Asteroids 2867 Steins and 21 Lutetia: surface composition from far infrared observations with the Spitzer space telescope
}

\author{
M. A. Barucci ${ }^{1}$, S. Fornasier ${ }^{1,2}$, E. Dotto ${ }^{3}$, P. L. Lamy ${ }^{4}$, L. Jorda ${ }^{4}$, O. Groussin ${ }^{4}$, J. R. Brucato ${ }^{5}$, \\ J. Carvano ${ }^{6}$, A. Alvarez-Candal ${ }^{1}$, D. Cruikshank ${ }^{7}$, and M. Fulchignoni ${ }^{1,2}$
}

${ }^{1}$ LESIA, Observatoire de Paris, 92195 Meudon Principal Cedex, France e-mail: antonella.barucci@obspm.fr

2 Université Paris Diderot, Paris VII, France

3 INAF, Osservatorio Astronomico di Roma, via Frascati 33, 00040 Monteporzio Catone, Roma, Italy

4 Laboratoire d'Astrophysique de Marseille, BP 8, 13376 Marseille Cedex 12, France

5 INAF, Osservatorio Astronomico di Capodimonte, via Moiariello 16, 80131 Napoli, Italy

6 Observatorio National (COAA), rua Gal. José Cristino 77, CEP20921-400 Rio de Janeiro, Brazil

7 NASA Ames Research Center, MS 245-6, Moffett Field, CA 94035-1000, USA

Received 14 June 2007 / Accepted 3 October 2007

\begin{abstract}
Aims. The aim of this paper is to investigate the surface composition of the two asteroids 21 Lutetia and 2867 Steins, targets of the Rosetta space mission.

Methods. We observed the two asteroids through their full rotational periods with the Infrared Spectrograph of the Spitzer Space Telescope to investigate the surface properties. The analysis of their thermal emission spectra was carried out to detect emissivity features that diagnose the surface composition.

Results. For both asteroids, the Christiansen peak, the Reststrahlen, and the Transparency features were detected. The thermal emissivity shows a clear analogy to carbonaceous chondrite meteorites, in particular to the CO-CV types for 21 Lutetia, while for 2867 Steins, already suggested as belonging to the E-type asteroids, the similarity to the enstatite achondrite meteorite is confirmed.
\end{abstract}

Key words. minor planets, asteroids - techniques: spectroscopic - methods: observational

\section{Introduction}

The Rosetta spacecraft was successfully launched on 2 March 2004 and is now on its way to rendez-vous with comet 67P/Churyumov-Gerasimenko in May 2014 at a heliocentric distance of 4.5 AU. During its journey to the comet, the spacecraft will fly by two main belt asteroids 2867 Steins and 21 Lutetia, selected after detailed studies of the candidates for all possible mission scenarios (Barucci et al. 2005). Nominally the fly-by of asteroid Steins will take place on 5 September 2008, at a velocity of $8.6 \mathrm{~km} \mathrm{~s}^{-1}$ and a closest approach of about $800 \mathrm{~km}$. That of Lutetia will take place on 10 June 2010 at a velocity of $15 \mathrm{~km} \mathrm{~s}^{-1}$ and a closest approach of about $3000 \mathrm{~km}$. As for all targets of space missions, an a priori knowledge of these terra incognita is crucial to preparing and optimizing the operations of the spacecraft and its instruments so as to maximize the scientific return.

We briefly summarize the properties of these two asteroids as presently known, based on the recent review article by Barucci et al. (2007). Asteroid 2867 Steins is a small asteroid whose knowledge is still limited. The first spectroscopic observations (Barucci et al. 2005) suggested a similarity between Steins and E-type asteroids, a rare class of objects with properties similar to the enstatite meteorites. Similar results have been obtained recently by Fornasier et al. (2007) and by Nedelcu et al. (2007a). Fornasier et al. (2006), using polarimetric technique, determined for the first time the albedo value of $0.45 \pm 0.10$, a high value that implies a diameter of approximately $4.6 \mathrm{~km}$ and confirms the E-type classification. Asteroid 21 Lutetia, a large object with a diameter of about $100 \mathrm{~km}$, has a V+NIR spectral behaviour similar to the carbonaceous chondrites (Birlan et al. 2004; Barucci et al. 2005; Birlan et al. 2006; Nedelcu et al. 2007b), but an IRAS albedo $(0.22 \pm 0.02$, Tedesco \& Veeder 1992) that is not compatible with C-type asteroids, which are associated to this type of meteorite. Instead, the IRAS albedo leads Lutetia to be classified as an M-type asteroid, suggesting a metallic composition. Nevertheless several different albedo values have been published. A lower albedo $0.09-0.10$ has been estimated from ground-based polarimetric measurements (Zellner et al. 1977; Lupisko \& Mohamed 1996), while a high value of $0.23 \pm 0.05$ was given by Mueller et al. (2006) with thermal-infrared observations. Other intermediate values of $0.15 \pm 0.05$ have been obtained by Magri et al. (1999) using a shape modelled by new radar observations, and $0.13 \pm 0.03$ by Carvano et al. (2007) analysing recent thermal-infrared observations.

We now report on far-infrared observations of these two asteroids carried out with the Infrared Spectrograph (IRS) of the Spitzer space telescope (SST) in a series of companion articles that allow us to discuss and try to solve some controversies before the Rosetta fly-bys. A detailed account of the observations, data processing, and results on the size, the albedo, and the thermal properties are presented in Lamy et al. (2007a,b). The present article analyses the emissivity spectra and discusses 
the mineralogical composition and the classification of the two Rosetta asteroid targets.

\section{Observations and data analysis}

2867 Steins and 21 Lutetia were observed with the IRS spectrograph which covers the wavelength range $5.2-38.0 \mu \mathrm{m}$ in four long-slit segments: the short wavelength, 2nd order (SL2, from 5.2 to $8.5 \mu \mathrm{m}$ ); the short wavelength, 1st order (SL1, from 7.4 to $14.2 \mu \mathrm{m}$ ); the long wavelength, 2nd order (LL2, from 14.0 to $21.5 \mu \mathrm{m}$ ); and the long wavelength, 1 st order (LL1, from 19.5 to $38.0 \mu \mathrm{m})$. The observations of 2867 Steins were carried out on 22 November 2005 from 06:23 to 13:18 UT, catching 14 full range $(5-38 \mu \mathrm{m})$, low resolution spectra that covered the asteroid' s full rotational period of $6.052 \pm 0.007 \mathrm{~h}$ (Kuppers et al. 2007). All the spectra were acquired with single ramps of $14.68 \mathrm{~s}$, except for the SL1 segment where we used a $6.29 \mathrm{~s}$ ramp. The observations were carried out when the asteroid was at heliocentric distance of 2.13 AU, 1.60 AU far away from SPITZER and seen with a phase angle of $27.2 \mathrm{deg}$.

Lutetia was observed from 17:32 UT on 10 December 2005 to $02: 27$ on 11 December 2005, obtaining 14 individual full wavelength range spectra covering the rotational period of $8.17 \pm$ $0.10 \mathrm{~h}$ (Zappalà et al. 1984). An exposure time of $6.29 \mathrm{~s}$ was used for each of the 4 spectral segments. The observations were carried out when the asteroid was at a heliocentric distance of 2.81 AU, 2.66 AU far away from Spitzer and seen with a phase angle of $21.1 \mathrm{deg}$.

Data reduction of the Basic Calibrated Data (BCD) was provided by the Spitzer pipeline (http://ssc.spitzer. caltech.edu/documents/SOM/irs60.pdf and http:// ssc.spitzer. caltech.edu/irs/dh/ IrsPDDmar30.pdf); background correction by differencing the 2 nodding positions was performed using SPICE, the Spitzer IRS Custom Extraction software. More details on the acquisition and data reduction steps are reported in Lamy et al. (2007a).

To interpret the infrared spectra, the thermal model (Groussin et al. 2004) was used and all the details are presented in Lamy et al. (2007a,b). The thermal balance on the surface includes the solar flux received by the object, on the one hand, the re-radiated flux and the heat conduction, on the other. The solar flux depends mainly on the albedo, the re-radiated flux on the beaming factor, and the heat conduction on the thermal inertia. We used a visible geometric albedo of $0.34 \pm 0.06$ for Steins and $0.19 \pm 0.02$ for Lutetia, a thermal inertia of $\sim 100 \mathrm{~J} / \mathrm{K} / \mathrm{m}^{2} / \mathrm{s}^{0.5}$ for Steins, and $25 \mathrm{~J} / \mathrm{K} / \mathrm{m}^{2} / \mathrm{s}^{0.5}$ for Lutetia, and a beaming factor $\eta=0.9$ for both asteroids, consistent with Lamy et al. (2007a,b).

Using the above parameters, we calculated the infrared spectral energy distribution (SED) of Steins and Lutetia. The SED is normalized to the SST data using a multiplicative coefficient, which corresponds to the change in cross-section as a function of time (light curve). Each spectrum observed by SST is then divided by the normalized SED to obtain the emissivity as a function of wavelength. The 14 individual emissivity spectra of 21 Lutetia and 2867 Steins are presented in Figs. 1 and 2.

We investigated the differences between the individual spectra for each asteroid by a $\chi$-squared value defined as:

$\chi^{2}=\sum_{i=1}^{N}\left(\frac{e_{\text {mean }}(i)-e(i)}{N}\right)^{2}$

where $N$ is the number of data points in the wavelength range 11-32 $\mu \mathrm{m}(N=196)$ for Steins, and 6-36 $\mu \mathrm{m}(N=331)$ for Lutetia, $e_{\text {mean }}$ the mean flux of the 14 different data sets and $e$ the emissivity of each individual data set. We report in Table 1 the computed residuals of each individual data set compared to the spectrum derived from the mean. The higher computed residual values for Steins are attributable to the lower signal to noise ratio for its spectral data. Considering the flux errors and the defects of the detector, we did not find any difference in the emissivity spectra of Lutetia and Steins during their rotational periods. Therefore, we take the mean of all the 14 spectra available for each object in order to increase the $\mathrm{S} / \mathrm{N}$ ratio, which, particularly in the case of 2867 Steins, was quite low in the SL2 range and for $\lambda>30 \mu \mathrm{m}$.

\section{Data interpretation}

To interpret the resulting emissivity of both targets in terms of their composition, we compared the mean emissivity (the average of the 14 observed spectra) with a wide sample of laboratory spectra of minerals and meteorites (Dotto et al. 2000, 2004; Barucci et al. 2002; Salisbury et al. 1991a,b; ASTER spectral library on http://speclib.jpl.nasa.gov; Relab database http://lf314-rlds.geo.brown.edu).

As thoroughly discussed by Salisbury (1993), the most diagnostic spectral characteristics in the analysed wavelength range are the Christiansen peak, the Reststrahlen, and Transparency features. The Christiansen peak is related to the mineralogy and grain size, and for silicates it occurs between 8 and $9.5 \mu \mathrm{m}$, just before the $\mathrm{Si}-\mathrm{O}$ stretching vibration bands. Reststrahlen bands are due to vibrational modes of molecular complexes, and for silicates they occur in the $8-25 \mu \mathrm{m}$ region. The Transparency features are volume scattering features of fine particulates, and they form troughs between the main Reststrahlen bands. At a small grain size, volume scattering occurs and Transparency features are observable due to a loss of photons crossing many grains.

The spectral behaviour of Lutetia, shown at the top of Fig. 3, appears to be consistent with that of carbonaceous chondrites, which exhibit the Christiansen peak at distinctively long wavelengths (Salisbury et al. 1991b). After analysing a large sample of meteorites taken from the RELAB and ASTER databases, we report in Fig. 3 a comparison with the carbonaceous chondrite Allende (CV type), which shows a very good spectroscopic match. The emissivity spectrum of the meteorite Odessa is also reported as an example. Odessa is an iron meteorite that contains some silicate inclusions. The spectral behaviour is completely different from that of Lutetia, so the possible metallic nature for this asteroid is rejected. A $10 \mu \mathrm{m}$ emission feature, together with a broad emissivity structure between about 16 and $28 \mu \mathrm{m}$, has been found in the emissivity spectra of Trojan asteroids observed with the Spitzer Space Telescope (Emery et al. 2006). They propose that the Trojan emission spectra may best be explained by a very underdense surface structure consisting of particles of just a few microns in size or by very small mineral and carbonaceous particles suspended in a matrix material that is relatively transparent in the mid-infrared. However, the detailed shape of the spectra and the $\mathrm{C}, \mathrm{R}$, and $\mathrm{T}$ features are different in the Trojans than in the data presented here.

To better investigate the primitive nature of 21 Lutetia and its similarity with carbonaceous chondrite meteorites, we compared the Spitzer spectrum with laboratory spectra of different samples at different grain sizes published by Barucci et al. (2002). The Lutetia emissivity spectrum matches very well the emissivity spectrum of Ornans meteorites, a CO3 type carbonaceous chondritic (Barucci et al. 2002), with grains in a size range smaller than $20 \mu \mathrm{m}$. In fact, the peak at about $9.3 \mu \mathrm{m}$ in the emissivity spectrum of Lutetia is consistent with the Christiansen 


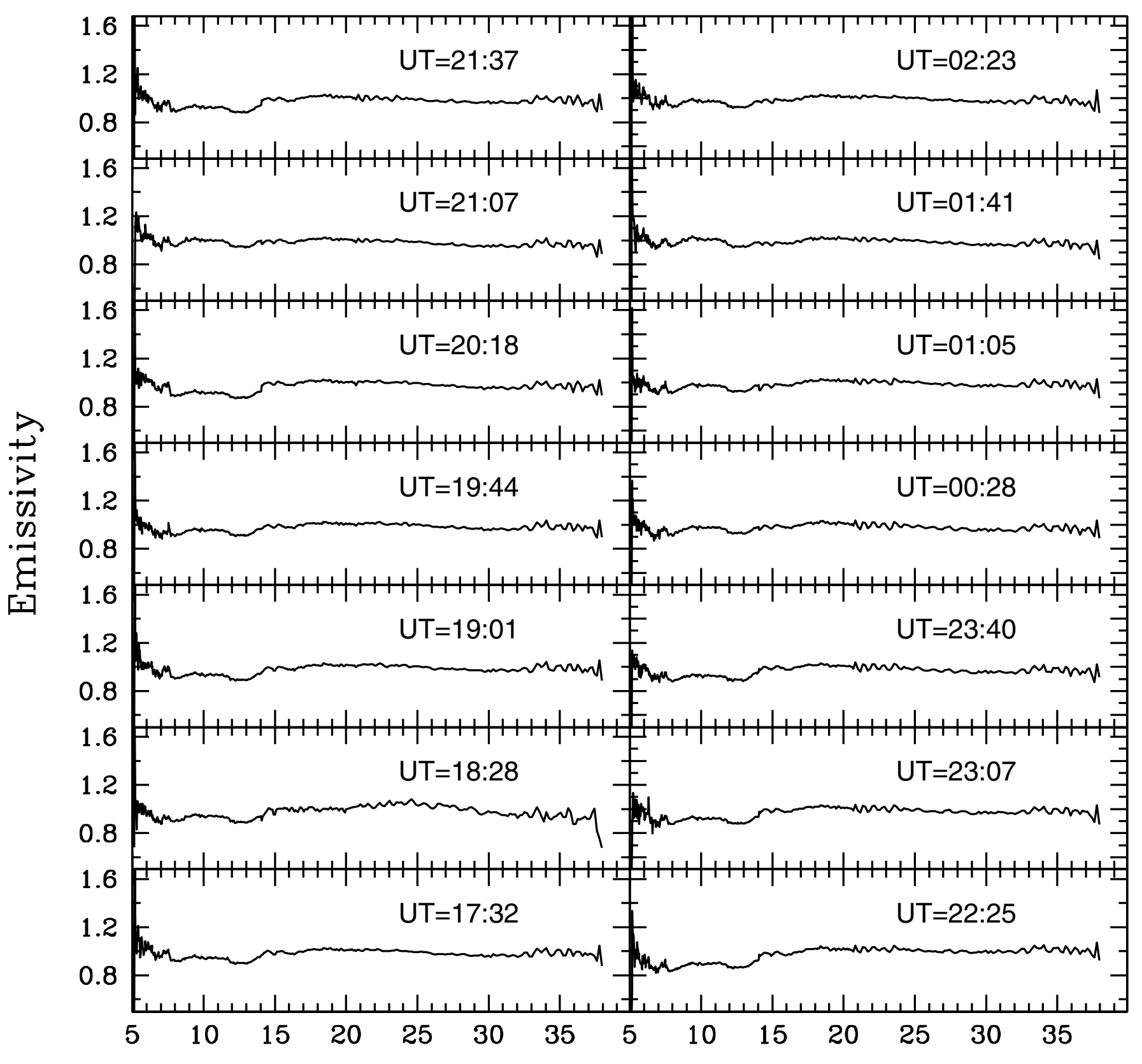

\section{Wavelength $(\mu \mathrm{m})$}

Fig. 1. Individual emissivity spectra of 21 Lutetia acquired on 10 December 2005 from 17:32 UT to 11 December 2006 02:27 UT (the UT time corresponds to the start of the exposure).

peak of the Ornans meteorite, and the plateau between 9 and $12 \mu \mathrm{m}$ is consistent with the Reststrahlen features, as well as the Transparency band around $13 \mu \mathrm{m}$. The match of the mid-infrared spectrum of Lutetia with that of Ornans, which suffered aqueous alteration processes (Zolensky \& McSween 1988), confirms the primitive chondritic character of this asteroid already proposed by Barucci et al. (2005) and Birlan et al. (2004, 2006) on the basis of ground-based observations. In fact, the visible and nearinfrared spectra are very similar to those of CO3 or CV3 type meteorites, with a stronger similarity to the Vigarano (CV3 type) meteorite.

Since few data in far-infrared region on asteroid analogue materials with different grain sizes exist in the literature, we performed new laboratory experiments for Vigarano using the Bruker IFS66v interferometer at the INAF Astronomical Capodimonte Observatory (Italy). We used the same equipment and the same conditions as was previously used to obtain the Ornans data (Barucci et al. 2002), and the results are reported in Fig. 4. Also, Vigarano with small grain size $(0-20 \mu \mathrm{m})$ fits the Lutetia spectrum well as shown by the correspondence of the Christiansen, Reststrahlen, and Transparency features. The Vigarano sample is characterised by the presence of aqueous alterations in its matrix. The close similarity to $\mathrm{CO} 3$ and $\mathrm{CV} 3$ meteorites containing hydrous mineral, suggests that Lutetia underwent some aqueous alteration. This result confirms what has already been inferred by Rivkin et al. (2000) by the detection 


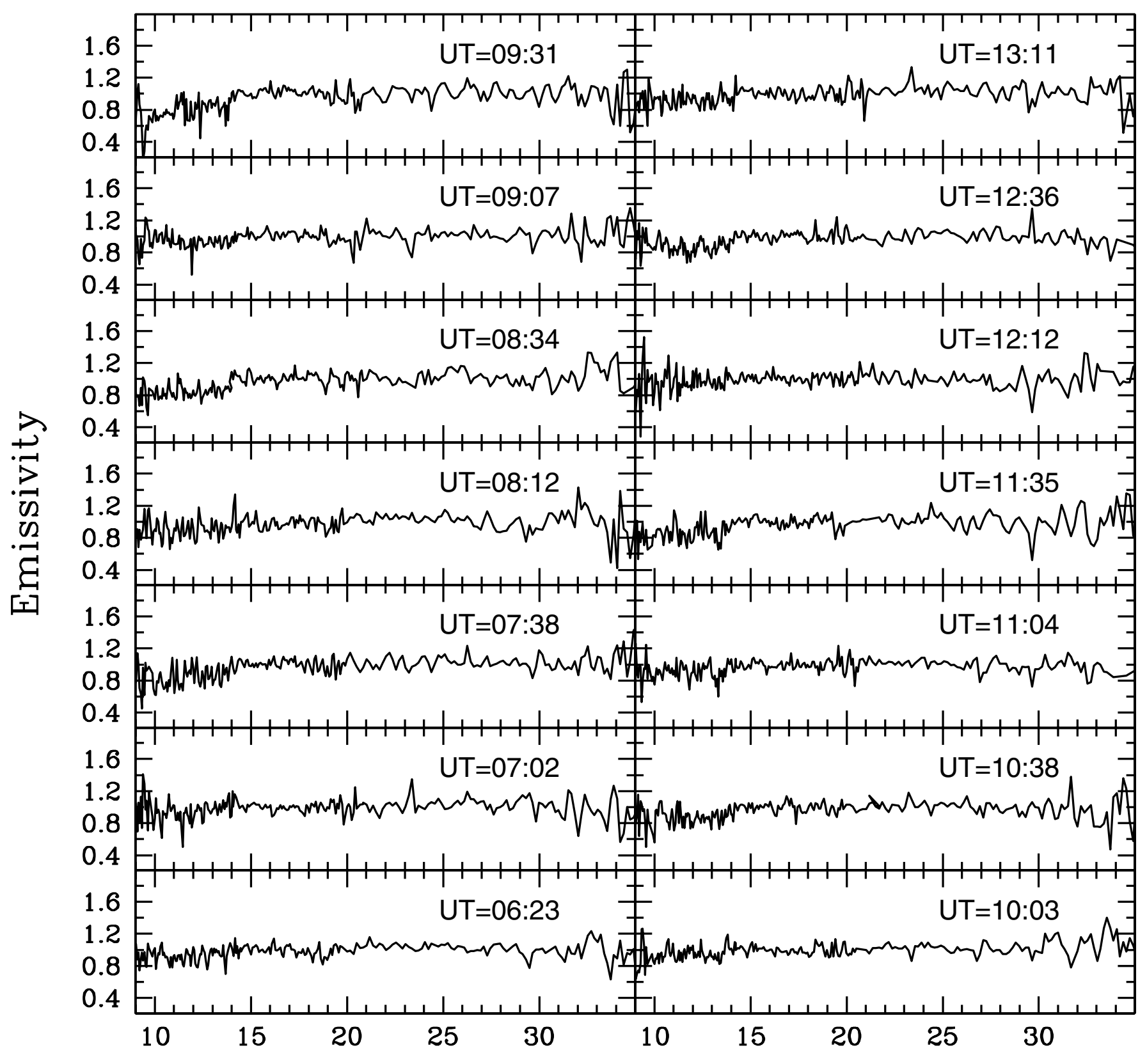

Wavelength $(\mu \mathrm{m})$

Fig. 2. Individual emissivity spectra of 2867 Steins acquired on 22 November 2005 from 06:23 to 13:11, UT time (the UT time corresponds to the start of the exposure).

of the $3 \mu \mathrm{m}$ absorption feature, a diagnostic for hydrated minerals. Birlan et al. (2006) also observed the $3 \mu \mathrm{m}$ band in the Lutetia spectrum, and they found a 2.9 vs. $3.2 \mu \mathrm{m}$ ratio value close to that of the CV-CO meteorites. Furthermore, Lazzarin et al. (2004) and Prokof'eva et al. (2005) obtained several visible specta and detected the possible presence of features at 0.44 and $0.67 \mu \mathrm{m}$, attributed to hydrated silicates.

Figure 5 shows the emissivity spectrum of Steins obtained with Spitzer as mean of all the 14 individual spectra. Although its signal is not precise enough to clearly distinguish the exact position of the different bands, the general behaviour of the spectrum and, in particular, the wavelength position of the Christiansen, Reststrahlen, and Transparency features, suggests a similarity to the aubrite (enstatite achondrite) meteorite and the enstatite mineral, a single-chain pyroxene of which the aubrite meteorites are primarily composed. In fact, as noted by Salisbury et al. (1991a), the aubrite spectrum exhibits the Christiansen peak at short wavelengths, around $8.3 \mu \mathrm{m}$, and strong Reststrahlen bands between 8.5 and $9.5 \mu \mathrm{m}$. Transparency features are evident around $12-13 \mu \mathrm{m}$. As a comparison, Fig. 5 also shows the emissivity spectrum of a sample of enstatite analysed and published by Barucci et al. (2002) and of the aubrite meteorite ALH84007 as taken from the ASTER library. The behaviour of our Spitzer mid-infrared spectrum and the match with laboratory spectra of the enstatite mineral and the aubrite meteorite support the classification of Steins given by 
Table 1. Residuals of each individual emissivity spectrum compared to the mean flux of the 14 spectra.

\begin{tabular}{ccc}
\hline \hline Set & Steins residuals & Lutetia residuals \\
\hline 1 & $2.40 \mathrm{e}-05$ & $8.22 \mathrm{e}-07$ \\
2 & $3.71 \mathrm{e}-05$ & $1.91 \mathrm{e}-06$ \\
3 & $3.30 \mathrm{e}-05$ & $5.71 \mathrm{e}-07$ \\
4 & $3.80 \mathrm{e}-05$ & $2.69 \mathrm{e}-07$ \\
5 & $2.71 \mathrm{e}-05$ & $1.38 \mathrm{e}-06$ \\
6 & $2.62 \mathrm{e}-05$ & $3.32 \mathrm{e}-06$ \\
7 & $4.51 \mathrm{e}-05$ & $6.40 \mathrm{e}-07$ \\
8 & $2.61 \mathrm{e}-05$ & $5.76 \mathrm{e}-06$ \\
9 & $2.54 \mathrm{e}-05$ & $1.58 \mathrm{e}-06$ \\
10 & $2.81 \mathrm{e}-05$ & $8.71 \mathrm{e}-07$ \\
11 & $4.24 \mathrm{e}-05$ & $9.29 \mathrm{e}-07$ \\
12 & $3.81 \mathrm{e}-05$ & $6.76 \mathrm{e}-07$ \\
13 & $2.74 \mathrm{e}-05$ & $3.21 \mathrm{e}-06$ \\
14 & $2.81 \mathrm{e}-05$ & $8.61 \mathrm{e}-07$ \\
\hline
\end{tabular}

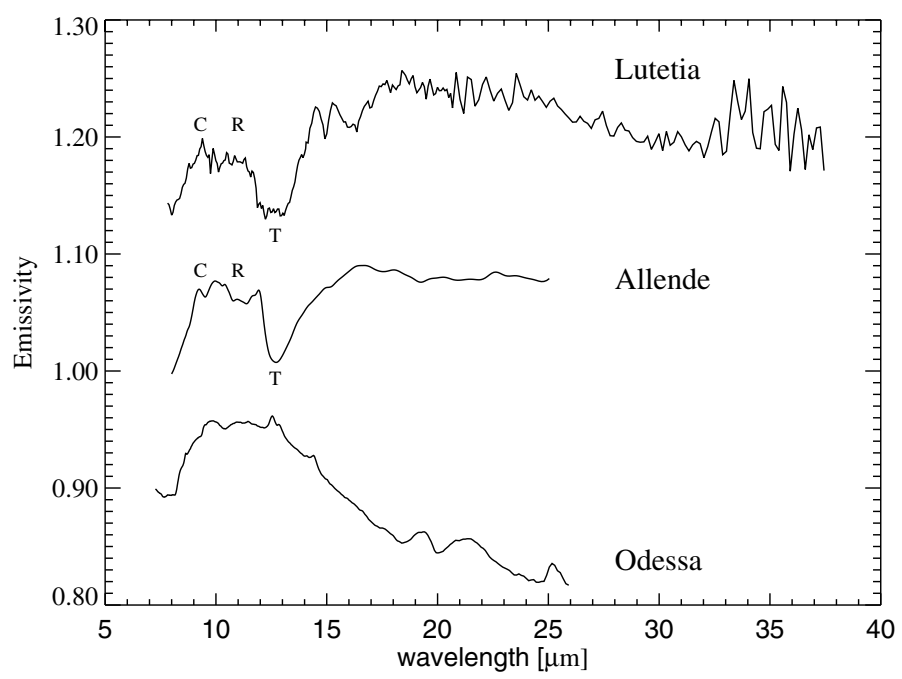

Fig. 3. The emissivity of Lutetia (average of the 14 individual spectra) compared with the emissivity of the Allende meteorite (from the ASTER database, with particle size $0-75 \mu \mathrm{m}$ ) and of the Odessa meteorite (from the RELAB database, with particle size 45-90 $\mu \mathrm{m}$ ). The error bars of the emissivity spectrum of Lutetia are $\sim 5 \%$ considering errors due both to the thermal data and to the model incertitude. The Christiansen, Reststrahlen and Transparency features are indicated as C, $\mathrm{R}$, and $\mathrm{T}$.

Barucci et al. (2005) as an E-type asteroid with an enstatite composition. This classification is also strengthened by the polarimetric properties (Fornasier et al. 2006).

\section{Conclusions}

21 Lutetia and 2867 Steins were observed with the Infrared Spectrograph (IRS) of the Spitzer Space Telescope. The emissivity spectra for each asteroid were derived after the division of the spectral energy distribution with a thermal model. To interpret the emissivity spectral features above the thermal emission continuum, we compared the Spitzer emissivity both with laboratory spectra available in the literature and with new laboratory experiments on the Vigarano meteorite at various grain sizes.

The Steins emissivity spectrum, even though it has low signal precision, is similar to the enstatite achondrite meteorites and to the enstatite mineral, confirming the rare E-type classification already suggested on the basis of ground-based spectral and polarimetric observations.

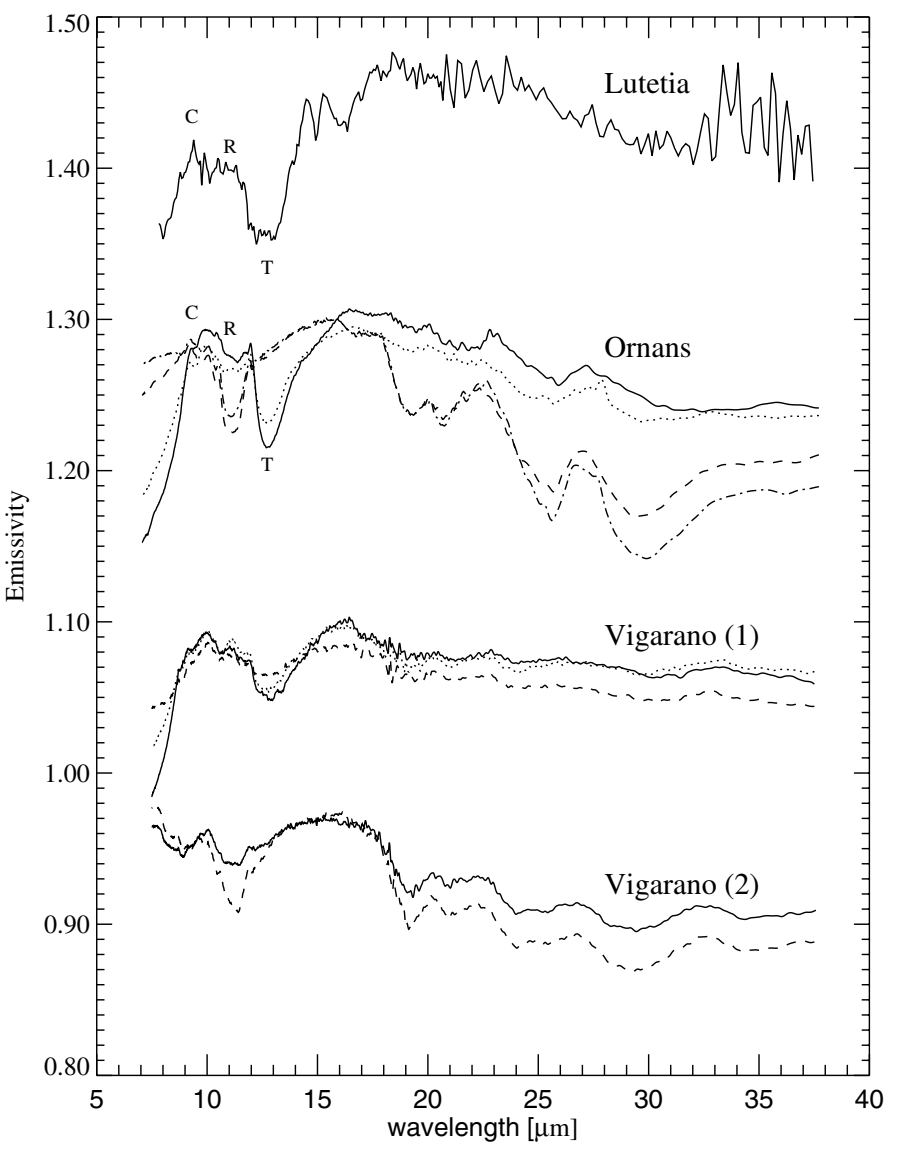

Fig. 4. The emissivity of Lutetia (average of the 14 individual spectra) compared with the emissivity of the Ornans meteorite by Barucci et al. (2005) at different grain dimensions $(0-20 \mu \mathrm{m}$, continuous line; 20-50 $\mu \mathrm{m}$, dotted line; 50-100 $\mu \mathrm{m}$, dashed line; and $>100 \mu \mathrm{m}$, dasheddotted line), and the emissivity of the Vigarano meteorite obtained with new laboratory experiments at different grain dimensions. Vigarano (1): continuous line for $0-20 \mu \mathrm{m}$; dotted line for $20-50 \mu \mathrm{m}$, and dashed line for 50-100 $\mu \mathrm{m}$; Vigarano (2): continuous line for $100-150 \mu \mathrm{m}$, and dashed line for grain size $>150 \mu \mathrm{m}$. All the spectra are shifted for clarity. The Christiansen, Reststrahlen, and Transparency features are indicated as $\mathrm{C}, \mathrm{R}$, and $\mathrm{T}$.

For 21 Lutetia, the emissivity spectrum departs significantly from the typical metallic meteorites, so that the first M classification derived from its high IRAS albedo is not confirmed. On the other hand, its emissivity in the $6-38 \mu \mathrm{m}$ range is similar to that of the $\mathrm{CO} 3$ and $\mathrm{CV} 3$ carbonaceous chondrites with a small grain size. Even though it is difficult to distinguish between the $\mathrm{CO}$ and $\mathrm{CV}$ spectra, the emissivity of the Lutetia spectrum seems more similar to that of Ornans with a smaller grain size $(0-20 \mu \mathrm{m})$, shown by the better correspondence of the Christiansen peak and the Restrahlen and Transparency features. The CO carbonaceous chondrites consist of small chondrules and aggregates set in a fine-grained matrix consisting of a heterogeneous mixture of fine-grained, iron-rich olivine and hydrated silicates (Sandford 1984). This similarity with the carbonaceous chondrites implies that Lutetia is a primordial body. Its surface has to be composed of particles of small size, with the possible presence of aqueous altered material that underwent slight thermal alteration.

The behaviour of the obtained emissivity data, in particular the broad analysed features are independent of albedo determination. 


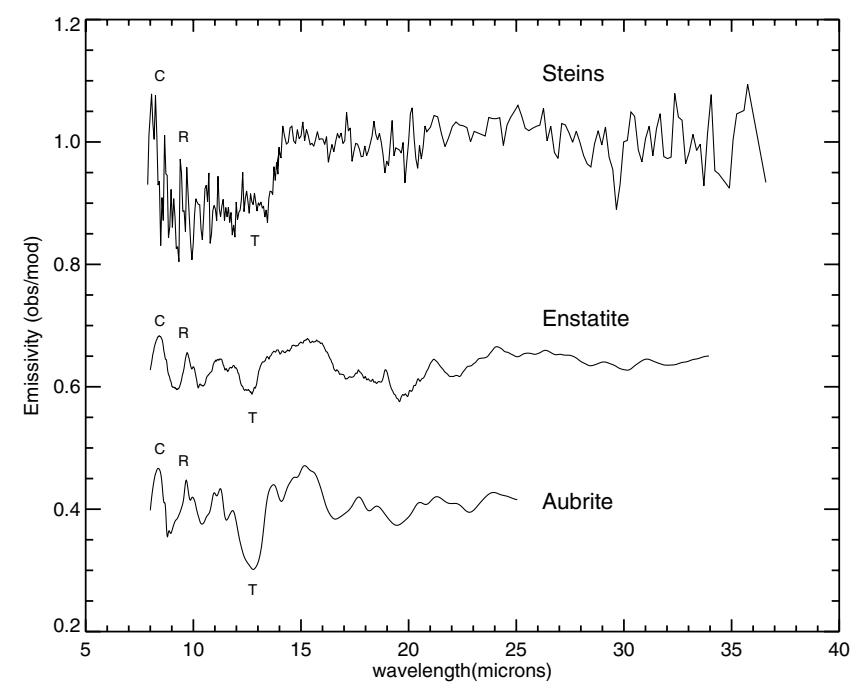

Fig. 5. The emissivity of Steins (average of the 14 individual spectra) compared with the emissivity of the aubrite meteorite ALH84007 taken from the ASTER database and that of the enstatite sample (with particle size 0-50 $\mu \mathrm{m}$ ) taken from Barucci et al. (2005). All the spectra are shifted for clarity. The Christiansen, Reststrahlen, and Transparency features are indicated as C, R, and T. The error bars of the emissivity spectrum of Steins are $\sim 11 \%$ considering both the errors due to the thermal data and to the model incertitude.

The albedo values of $0.19 \pm 0.02$ (Lamy et al. 2007b) derived from the Spitzer data analysis using an $H_{V}=7.3$, is too high with respect to the average albedo value of $\mathrm{CO}$ carbonaceous chondrite albedo. The Lutetia albedo still remains a puzzle point, as discussed by Carvano et al. (2007). Some high albedo particle inclusions that do not influence the spectral reflectivity behaviour could be the origin of the discrepancy in the albedo values.

In the case of Steins, the obtained albedo of $0.34 \pm 0.06$ (Lamy et al. 2007a) agrees with the typical values of E-type asteroids. The incertitude on the $G$ parameter value $(G=0.35 \pm$ $0.05)$, due to poor sampling of the phase curve at small phase angles, and the consequent estimation of the absolute magnitude $H_{V}=13.34 \pm 0.07$ (Jorda et al. 2007), could be the cause of the small variation in the albedo determination.
Acknowledgements. This work is based on observations made with the Spitzer Space Telescope, which is operated by the Jet Propulsion Laboratory, California Institute of Technology under a contract with NASA.

\section{References}

Barucci, M. A., Dotto, E., Brucato, J. R., et al. 2002, Icarus, 156, 202 Barucci, M. A., Fulchignoni, M., Fornasier, S., et al. 2005, A\&A, 430, 313 Barucci, M. A., Fulchignoni, M., \& Rossi, A. 2007, SSR, 128, 67 Birlan, M., Barucci, M. A., Vernazza, P., et al. 2004, New Astron., 9, 343 Birlan, M., Vernazza, P., Fulchignoni, M., et al. 2006, A\&A, 454, 677 Carvano, J. M., Barucci, M. A., Fornasier, S., et al. 2007, A\&A, in press Dotto, E., Mueller, T. G., Barucci, M. A., et al. 2000, A\&A, 358, 1133 Dotto, E., Barucci, M. A., Brucato, J. R., et al. 2004, A\&A, 427, 1081 Emery, J., Cruikshank, D. P., \& Van Cleve, J. 2006, Icarus, 182, 496 Fornasier, S., Belskaya, I., Fulchignoni, M., et al. 2006, A\&A, 449, 9 Fornasier, S., Marzari, F., Dotto, E., Barucci, M. A., \& Migliorini, A. 2007, A\&A, 474, L29

Groussin, O., Lamy, P., \& Jorda, L. 2004, A\&A, 413, 1163

Jorda, L., Lamy, P., Faury, G., et al. 2007, A\&A, submitted

Kuppers, M., Mottola, S., Lowry, S., et al. 2007, A\&A, 462, L13 Lamy, P., Jorda, L., Fornasier, S., et al. 2007a, A\&A, in preparation Lamy, P., Kaasalainen, M., Weissman, P., et al. 2007b, A\&A, in preparation Lazzarin, M., Marchi, S., Magrin, S., \& Barbieri, C. 2004, A\&A, 425, L25 Lupishko, D. F., \& Mohamed, R. A. 1996, Icarus, 140, 209

Magri, C., Ostro, S. J., Rosema, K. D., et al. 1999, Icarus, 140, 379

Mueller, M., Harris, A. W., Bus, S. J., et al. 2006, A\&A, 447, 1153

Nedelcu, D. A., Birlan, M., \& Vernazza, P. 2007a, A\&A, 473, L33

Nedelcu, D. A., Birlan, M., \& Vernazza, P. 2007b, A\&A, 470, 1157

Prokof'eva, V. V., Bochkov, V. V., \& Buserev, V. V. 2005, Solar System Research, 39(5), 410

Rivkin, A. S., Howell, E. S., Lebofsky, L. A., et al. 2000, Icarus, 145, 351

Salisbury, J. W., D’Aria, D. M., \& Jarosewich, E. 1991a, Icarus, 92, 280

Salisbury, J. W., Walter, L. S., Vergo, N., \& D'Aria, D. M. 1991b, Infrared (2.1-25 micron) Spectra of Minerals (Baltimore: Johns Hopkins Press)

Salisbury, J. M. 1993, in Remote Geochemical Analysis: Elemental and Mineralogical Composition, ed. C. M. Pieters, \& P. A. J. Englert (Cambridge: Cambridge Univ. Press), 79

Sandford, S. 1984, Icarus, 60, 115

Tedesco, E. F., \& Veeder, G. J. 1992, in The IRAS Minor Planet Survey, ed. E. F. Tedesco, G. J. Veeder, J. W. Fowler, \& J. R. Chillemi, Technical Report PL-TR-92-2049, Phillips Laboratory, Hanscom AF Base, MA

Zappalà, V., Di Martino, M., Knezevic, Z., \& Djurasevic, G. 1984, A\&A, 130, 208

Zolensky, M., \& McSween, H. Y. 1988, in Meteorites and the Early Solar System, ed. J. F. Kerridge, \& M. S. Matthews (Tucson: Univ. of Arizona Press), 1139

Zellner, B., Leake, M., LeBerte, T., et al. 1977, Proceedings of the Lunar Science Conference, 8th Ed. (Oxford: Pergamon Press), 1091 